\title{
High incidence of prostate cancer metastasis in Afro-Brazilian men with low educational levels: a retrospective observational study
}

\author{
Alexandre Barbosa Câmara de Souza', Hugo Gonçalo Guedes ${ }^{1}$, Victor Carbone Bernardes Oliveira', \\ Fábio Aires de Araújo ${ }^{1}$, Carlos Cesar Oliveira Ramos², Karina Carla Paula Medeiros ${ }^{1}$ \\ and Raimundo Fernandes Araújo Jr ${ }^{\text {** }}$
}

\begin{abstract}
Background: This study investigated factors related to ethnicity and educational level, their correlation with tumor stage at the time of diagnosis, and their influence on treatment outcomes in patients with prostate cancer.

Methods: In this retrospective observational study, we analyzed the medical records of 1,349 male patients treated for prostatic adenocarcinoma. We collected information about sociodemographic variables, including educational level and self-reported skin color. We also classified the disease according whether it was to more likely to present with metastasis and measured the tumor response to treatment.
\end{abstract}

Results: Less-educated ( $<8$ years of education) individuals were 4.8 times more likely to develop metastasis than those with more education ( $>11$ years of education; $p<0.001$ ). Similarly, patients with a self-reported black skin color had a $300 \%$ increased risk of metastasis at diagnosis $(p=0.001)$. Distant metastasis was independently correlated with worse outcomes, such that individuals with distant metastasis were 10 times more likely to die than were those without distant metastasis.

Conclusions: Patients with self-reported black skin color and $<8$ years of education were more likely to display advanced disease at the time of diagnosis compared with their counterparts. Only the presence of metastasis was independently associated with mortality or progressive disease.

Keywords: Epidemiology, Prognosis, Prostate cancer, Risk factor, Educational levels

\section{Background}

Prostate adenocarcinoma is the sixth most prevalent cancer in the world and the most common cancer among men in Europe, North America, and parts of Africa. After non-melanoma skin cancer, prostate adenocarcinoma is the second most often diagnosed cancer and the third leading cause of cancer-related deaths in the United States (US) [1]. In addition, prostate adenocarcinoma is the second most prevalent cancer in Brazil, with an estimated 60,180 new cases in 2012. According to the National Cancer Institute of Brazil, the increase in incidence rates may be explained by increased life expectancy, improvement

\footnotetext{
*Correspondence: araujojr@cb.ufrn.br

'Department of Morphology, Federal University of Rio Grande do Norte, Natal 59072-970, Rio Grande do Norte, Brazil

Full list of author information is available at the end of the article
}

in and development of diagnostic methods, and improved quality of information systems in Brazil [2].

There are differences in prostate adenocarcinoma rates across ethnic groups. In the US, African-Americans have the highest disease rate compared with other ethnicities. Caucasians have an approximately $60 \%$ lower rate of prostate adenocarcinoma diagnosis than do African-Americans, and the prevalence is even lower among those with a Latin American or Asian origin [3]. Differential genetic susceptibility and socioeconomic and environmental factors likely contribute to the observed differences in incidence rates among ethnicities [4-7]. For example, obesity is one of the most studied factors in cancer. Obesity has been associated with increased exposure to carcinogens in the diet [8], increased cardiovascular risk [9], higher prevalence of high-grade prostate cancer

\section{Biomed Central}


(15.5\%), and increased patient morbidity and mortality [10-13]. Thus, several factors are involved in prostate adenocarcinoma epidemiology, and the identification of useful prognostic factors for this neoplasm is extremely important for understanding the disease.

The most important clinical factors currently available are the prostate-specific antigen (PSA) level and histological differentiation degree in tumor biopsy fragments; these two markers are expressed as the Gleason score $[14,15]$. Although the Gleason score is valuable, its sole use does not define patient prognosis for prostate cancer [16]. Consequently, additional comprehensive studies of prognostic factors for prostatic adenocarcinoma are necessary to find the population that is most in need of attention and early diagnosis.

This study aimed to identify ethnic and educational levels as additional factors related to prostate adenocarcinoma, determine their correlation with tumor stage at the time of diagnosis, and elucidate their influence on patient outcomes.

\section{Methods}

The institutional committees (No. 030/0030/2006) of the Liga Norteriograndense Contra o Cancer, Brazil, approved this research.

This transversal study included the retrospective analysis of mortality or progressive disease state in 1,349 male patients with biopsy-confirmed prostate adenocarcinoma (International Classification of Diseases, 10th edition, code C61). Patients were treated between 1997 and 2005 at Luis Antonio Hospital, a Brazilian Health System cancer referral center in Natal, Brazil. Natal is the capital of Rio Grande do Norte State in the northeastern part of the country and has a population of $>1$ million inhabitants. Trained researchers collected data, which included hospital and medical records, between August 2007 and May 2008.

From all cases, researchers gathered medical record data related to the variables of interest: patient age at cancer diagnosis, race/ethnicity, residential address at diagnosis, tumor stage and grade, deaths, age, and educational level $(<8$ years $=$ incomplete elementary school; $>11$ years $=$ high school completion). Race/ethnicity was classified according to the mutually exclusive self-report for skin color (black: yes or no). After diagnosis, we defined the disease as either aggressive or nonaggressive. Regional or distant tumors based on imaging study results were classified as aggressive, while tumors confined to the prostate (localized) were nonaggressive. The endpoint was mortality or progressive disease at the end of the first stage of treatment (first consultation after hospital treatment, with 2 weeks to 1 month).

A chi-squared test determined the distribution differences of educational status and skin color. Multivariate logistic regression models examined the influence of socioeconomic factors on the presence of metastasis at diagnosis and patient outcome after the first stage of treatment. Regression analyses were conducted in two steps: Model 1 assessed the correlation of each socioeconomic variable with the presence or absence of metastasis at diagnosis, and Model 2 adjusted education status and skin color for the presence of metastasis to determine the impact of these variables on mortality. The analyses showed no colinearity. All statistics were performed using SPSS software (ver. 13.0 for Windows; SPSS Inc., Chicago, IL, USA) with a significance level of $p<0.05$.

\section{Results}

Table 1 shows the descriptive data of the study participants.

Univariate analysis showed a significant correlation between the presence of aggressive metastasis and educational levels $(p<0.001)$ and self-reported skin color $(p=0.006)$, suggesting that these variables are risk factors for distant metastasis at the time of diagnosis.

Table 1 Descriptive data

\begin{tabular}{|c|c|c|c|c|}
\hline Variable & $n$ & $\%$ & Mean & SD \\
\hline Age (years) & & & 71.25 & 9.10 \\
\hline$\leq 50$ & 23 & 1.7 & & \\
\hline $51-60$ & 151 & 11.2 & & \\
\hline $61-70$ & 401 & 29.7 & & \\
\hline $71-80$ & 562 & 41.7 & & \\
\hline $81-90$ & 205 & 15.2 & & \\
\hline$>90$ & 7 & 0.5 & & \\
\hline Educational level (years) ${ }^{\Xi}$ & & & - & - \\
\hline$<8$ & 504 & 66.9 & & \\
\hline$>11$ & 249 & 33.1 & & \\
\hline Self-reported skin color ${ }^{\Xi}$ & & & - & - \\
\hline Black & 122 & 11.9 & & \\
\hline Other & 905 & 88.1 & & \\
\hline Treatment $^{\Xi}$ & & & - & - \\
\hline Surgery & 190 & 14.9 & & \\
\hline Radiotherapy & 294 & 23.0 & & \\
\hline Chemotherapy & 26 & 2.0 & & \\
\hline Hormone therapy & 512 & 40.1 & & \\
\hline Multiple treatments & 254 & 19.9 & & \\
\hline Metastasis ${ }^{\Xi}$ & & & - & - \\
\hline Yes & 272 & 24.3 & & \\
\hline No & 849 & 75.7 & & \\
\hline Mortality or progressive disease $\Xi$ & & & - & - \\
\hline Yes & 608 & 77.9 & & \\
\hline No & 172 & 22.1 & & \\
\hline
\end{tabular}

$S D$ standard deviation.

$\Xi_{\text {categorical variables. }}$ 
Multivariate regression analysis confirmed these results and demonstrated independent correlations: the chance of developing an aggressive metastasis was increased 4.8-fold in patients with $<8$ years of education compared with those who had received $>11$ years of education $(p<0.001)$. Moreover, patients with a self-reported black skin color showed a $300 \%$ increase in aggressive metastasis compared with patients of other skin colors $(p=0.001)$.

Although self-reported skin color was not associated with mortality or progressive disease, low educational levels $(p=0.002)$ and the presence of metastasis $(p<0.001)$ were positively associated with the endpoint. The results from the multivariate regression analyses demonstrated that only the presence of aggressive metastasis was independently correlated with a negative outcome, such that patients with distant metastasis were 10 times more likely to die (Table 2).

\section{Discussion}

The inaccurate prediction of treatment outcomes for patients with prostate cancer is a public health problem because different treatment options are associated with different morbidity rates. For example, erectile dysfunction affects $80 \%$ of patients who have undergone radical prostatectomy [16]. The identification of additional prognostic factors will thus help physicians select the most appropriate treatment for each case.

Our results that a lower educational level was related to an increased likelihood of having a metastasis at the time of diagnosis may be due to decreased healthcare use among less-educated patients. Individuals who do not seek treatment or do not have access to healthcare may receive a delayed diagnosis and be less likely to adhere to treatment regimens. In contrast, well-educated individuals are more likely to undergo timely screening (PSA testing) and urological monitoring. A study conducted in Sao Paulo State in Brazil found that 44\% of 992 men with a low socioeconomic status had never received a preventive examination for prostate adenocarcinoma; this pattern was most prevalent among men aged $<70$ years, those with a black or brown skin color, and those with $<9$ years of education [17]. Another study in Sao Paulo showed high mortality rates among men living in poor areas, which may be related to a lack of access to preventive cancer screening [18]. In addition, a significant association between low educational level and prostate cancer incidence was demonstrated in a cohort from a US study. Although these subjects were followed for an average of 8.2 years, a strong association with cancer dissemination was not found [19].

Individuals with African descent have been associated with higher prostate cancer incidence rates and worse prognoses compared with individuals of Caucasian ethnicity. A United Kingdom study found that the incidence of prostate cancer was 166/100,000 among black men (black Caribbean, black Africans, and black unclassified), but only 56.4/100,000 among white men [20]. This difference in incidence rates has also been documented in the US, where African-American men show a higher incidence of prostate cancer, have a 2-fold greater likelihood of future disease development, and display twice the mortality risk compared with Caucasian men $[21,22]$.

Based upon previous findings and our current results, African descent and a low educational level may have interacting effects on prostate cancer treatment outcomes. In several countries throughout the world, individuals of African descent receive less education than do other ethnic groups, such as Caucasians; however, studies have shown that factors related to the lack of education alone cannot explain the treatment outcomes associated with this group. It is possible that genetic factors inherent to this population, including the expression of genes related to androgen activity, affect the presentation of prostate cancer. For example, young men of African descent have higher testosterone levels and exhibit changes in androgen receptors. These factors may account for the higher

Table 2 Multivariate regression analyses

\begin{tabular}{|c|c|c|c|c|c|c|}
\hline & & & Metastasis & & & Mortality or progressive disease \\
\hline & Yes (\%) & $p$ & OR $(95 \% \mathrm{Cl})$ & Yes (\%) & $p$ & OR $(95 \% \mathrm{Cl})$ \\
\hline Educational level (years) & & $<0.001$ & $4.778(2.821-8.093)$ & & 0.148 & \\
\hline$<8$ & $104(25.2)$ & & & $194(6.7)$ & & \\
\hline$>11$ & $24(10.4)$ & & & $124(89.2)$ & & \\
\hline Self-reported skin color & & 0.001 & $3.053(1.552-6.004)$ & & - & - \\
\hline Black & $15(13.4)$ & & & $72(84.7)$ & & - \\
\hline Other & $190(25.2)$ & & & $428(79.3)$ & & \\
\hline Metastasis & & - & - & & $<0.001$ & $10.026(6.187-16.247)$ \\
\hline Yes & $272(100.0)$ & & - & $80(44.0)$ & & \\
\hline No & $0(0.0)$ & & & $489(90.6)$ & & \\
\hline
\end{tabular}

$O R$ odds ratio, $\mathrm{Cl}$ confidence interval. 
incidence of prostate cancer and worse treatment outcomes observed in this group, excluding the impact of socioeconomic effects $[23,24]$. Therefore, while educational level and genetic predispositions influence the development and treatment of prostate cancer in individuals of African ethnicity, the interaction between these variables may have an even greater impact on disease outcomes.

We acknowledge that this study has limitations. First, this was a retrospective study, and potential confounding effects of complex variable relationships (i.e., PSA screening, Gleason score, and number of prior physician visits) may have limited our power of analysis. Unfortunately, these variables could not be analyzed. Second, there may have been variations or inaccuracies in patient self-reported information, which may have biased our results. However, we believe that the large sample size likely negated, or at least minimized, possible bias.

\section{Conclusions}

This study demonstrated that men of African descent and those with $<8$ years of education had a greater chance of displaying advanced disease at the time of diagnosis compared with patients of other skin colors and higher educational level ( $>11$ years). These findings should guide improvements in the public health system to change these patterns. Our results show that this population deserves special attention to ensure early diagnosis and treatment as well as improved health outcomes. Further research should be conducted to better understand these variables, other related factors, and the relationships among genetic and sociodemographic influences to improve prognostic classification in the Brazilian population.

\section{Competing interests}

The authors declare that they have no competing interests.

\section{Authors' contributions}

$\mathrm{ABCS}$ and HGG participated in the study design, performed statistical analyses, and drafted the manuscript. VCBO and FAA participated in the study design and drafted the manuscript. CCOR conducted the prostate cancer biopsies. KCPM helped design the study. RFAJ conceived the study, participated in the study design, and coordinated and drafted the manuscript. All authors read and approved the final manuscript.

\section{Authors' information \\ ABCS, HGG, VCBO and FAA are Medicine Doctors graduates of the Federa University of Rio Grande do Norte; CCOR is Pathologist of the Doctor Luiz Antonio Hospital; KCPM is Professor of Biomedicine of the Federal University of Rio Grande do Norte and RFAJ is PhD, Professor of Medicine of the, Federal University of Rio Grande do Norte.}

\section{Author details}

${ }^{1}$ Department of Morphology, Federal University of Rio Grande do Norte, Natal 59072-970, Rio Grande do Norte, Brazil. Doctor Luiz Antonio Hospital, Natal 59040-000, Rio Grande do Norte, Brazil.

\section{References}

1. Guedes HG, de Souza ABC, de Oliveira VCB, Araújo FA, Araújo Júnior RF: Epidemiological profile of prostate cancer around the world. REUOL 2008, 1(4):347-352.

2. Brasil: Estimative/2012 - Cancer Incidence in Brazil. Rio de Janeiro: INCA; 2011:122.

3. Delongchamps NB, Singh A, Haas GP: Epidemiology of prostate cancer in Africa: another step in the understanding of the disease? Curr Probl Cancer 2007, 31(3):226-236.

4. Martin NE, Mucci LA, Loda M, Depinho RA: Prognostic determinants in prostate cancer. Cancer J 2011, 17(6):429-437.

5. Platz EA, Leitzmann MF, Michaud DS, Willett WC, Giovannucci E: Interrelation of energy intake, body size, and physical activity with prostate cancer in a large prospective cohort study. Cancer Res 2003, 63(23):8542-8548.

6. Leavy J, Ambrosini G, Fritschi L: Vietnam military service history and prostate cancer. BMC Public Health 2006, 6:75.

7. Ruzzin J: Public health concern behind the exposure to persistent organic pollutants and the risk of metabolic diseases. BMC Public Health 2012, 12:298.

8. Mostaghel EA, Solomon KR, Pelton K, Freeman MR, Montgomery RB: Impact of circulating cholesterol levels on growth and intratumoral androgen concentration of prostate tumors. PLoS One 2012, 7(1):e30062.

9. de Lima JG, Nobrega LH, de Souza AB: Body adiposity index indicates only total adiposity, not risk. Obesity (Silver Spring) 2012, 20(6):1140.

10. Fowke JH, Motley SS, Concepcion RS, Penson DF, Barocas DA: Obesity, body composition, and prostate cancer. BMC Cancer 2012, 12:23.

11. Sarma AV, Jaffe CA, Schottenfeld D, Dunn R, Montie JE, Cooney KA, et al: Insulin-like growth factor-1, insulin-like growth factor binding protein-3, and body mass index: clinical correlates of prostate volume among Black men. Urology 2002, 59(3):362-367.

12. Conde FA, Sarna L, Oka RK, Vredevoe DL, Rettig MB, Aronson WJ: Age, body mass index, and serum prostate-specific antigen correlate with bone loss in men with prostate cancer not receiving androgen deprivation therapy. Urology 2004, 64(2):335-340.

13. Rogers LQ, Courneya KS, Paragi-Gururaja R, Markwell SJ, Imeokparia R: Lifestyle behaviors, obesity, and perceived health among men with and without a diagnosis of prostate cancer: a population-based, cross-sectional study. BMC Public Health 2008, 8:23.

14. Migowski A, Silva GA: Survival and prognostic factors of patients with clinically localized prostate cancer. Rev Saude Publica 2010, 44(2):344-352.

15. Gleason DF, Mellinger GT: Prediction of prognosis for prostatic adenocarcinoma by combined histological grading and clinical staging. 1974. J Urol 2002, 16(2 Pt 2):953-958. discussion 59.

16. Platz EA, Pollak MN, Leitzmann MF, Stampfer MJ, Willett WC, Giovannucci E: Plasma insulin-like growth factor- 1 and binding protein-3 and subsequent risk of prostate cancer in the PSA era. Cancer Causes Control 2005, 16(3):255-262.

17. Amorim VM, Barros MB, Cesar CL, Goldbaum M, Carandina L, Alves MC: Factors associated with prostate cancer screening: a population-based study. Cad Saude Publica 2011, 27(2):347-356.

18. Belon AP, Barros MB, Marin-Leon L: Mortality among adults: gender and socioeconomic differences in a Brazilian city. BMC Public Health 2012, 12:39.

19. Mouw T, Koster A, Wright ME, Blank MM, Moore SC, Hollenbeck A, et al: Education and risk of cancer in a large cohort of men and women in the United States. PLoS One 2008, 3(11):e3639.

20. Ben-Shlomo Y, Evans S, Ibrahim F, Patel B, Anson K, Chinegwundoh F, et al: The risk of prostate cancer amongst black men in the United Kingdom: the PROCESS cohort study. Eur Urol 2008, 53(1):99-105.

21. Jemal A, Murray T, Samuels A, Ghafoor A, Ward E, Thun MJ: Cancer statistics, 2003. CA Cancer J Clin 2003, 53(1):5-26.

22. Hoffman RM, Gilliland FD, Eley JW, Harlan LC, Stephenson RA, Stanford JL, et al: Racial and ethnic differences in advanced-stage prostate cancer: the Prostate Cancer Outcomes Study. J Nat/ Cancer Inst 2001, 93(5):388-395.

23. Gapstur SM, Gann PH, Kopp P, Colangelo L, Longcope C, Liu K: Serum androgen concentrations in young men: a longitudinal analysis of associations with age, obesity, and race. The CARDIA male hormone study. Cancer Epidemiol Biomarkers Prev 2002, 11 (10 Pt 1):1041-1047.

24. Freedland SJ, Isaacs WB: Explaining racial differences in prostate cancer in the United States: sociology or biology? Prostate 2005, 62(3):243-252.

doi:10.1186/1471-2458-13-537

Cite this article as: de Souza et al:: High incidence of prostate cancer metastasis in Afro-Brazilian men with low educational levels: a retrospective observational study. BMC Public Health 2013 13:537. 